\title{
New Way to Calculate Ricci Tensor and Ricci Scalar
}

\author{
Abed El Karim S. Abou Layla \\ Independent Researcher, Gaza City, Palestine \\ Email: a.k.aboulayla@gmail.com
}

How to cite this paper: Abou Layla, A.K. (2019) New Way to Calculate Ricci Tensor and Ricci Scalar. Journal of High Energy Physics, Gravitation and Cosmology, 5, 850-867.

https://doi.org/10.4236/jhepgc.2019.53042

Received: June 12, 2019

Accepted: July 12, 2019

Published: July 15, 2019

Copyright $\odot 2019$ by author(s) and Scientific Research Publishing Inc. This work is licensed under the Creative Commons Attribution International License (CC BY 4.0).

http://creativecommons.org/licenses/by/4.0/

cc) (i) Open Access

\begin{abstract}
In the theory of general relativity, the finding of the Einstein Field Equation happens in a complex mathematical operation, a process we don't need any more. Through a new theory in vector analysis, we'll see that we can calculate the components of the Ricci tensor, Ricci scalar, and Einstein Field Equation directly in an easy way without the need to use general relativity theory hypotheses, principles, and symbols. Formulating the general relativity theory through another theory will make it easier to understand this relativity theory and will help combining it with electromagnetic theory and quantum mechanics easily.
\end{abstract}

\section{Keywords}

General Relativity, Ricci Tensor, Ricci Scalar, Einstein Field Equation, Stress-Energy Tensor, Robertson-Walker Metric, Schwarzschild Metric

\section{Introduction}

All the books that explain general relativity theory present it in a conventional and complex way, an issue made it impossible to unit this theory with the electromagnetic theory and the quantum mechanics.

I resolved this problem through redefining the relativity theory in a different way and tie it with a new theory in the vector analysis; at present time I publish my research papers in this theory leading to unify the three theories in one.

To define my new theory (called the extended field theory), I published my first research papers, Old Mechanics, Gravity, Electromagnetics and Relativity in One Theory: "Part I", in "Journal of High Energy Physics, Gravitation and Cosmology (JHEPGC)", I implemented some principles and derived some of physics equations that can apply to both electric and gravitational fields, without distinguishing in this theory. 
My second research published in the same journal titled "General relativity without Space-Time ( $\mathrm{G} \mathrm{R})$ " in which, I calculated the stress-energy tensor of the electric and gravitational fields in General relativity theory without the need of curved Space-Time concept.

In this research, we will calculate the components of the Ricci tensor and Ricci scalar for many metrics, such as The Robertson-Walker metric and Schwarzschild metric directly, means that we don't need the hypotheses, principles, and symbols of general relativity theory to get stress energy tensor, and this is important to annul the difference between the electromagnetic and gravitational, to better understanding of the universe, gravity, and black holes.

We still have to point that there is a general state to calculate the components of the Ricci tensor and Ricci scalar, which I will publish in next researches with all proofs and details.

\section{Basic Notions}

\subsection{The Equation of Interval Angular Vector}

Let's assume the orthonormal vector $z$ as the following

$$
\boldsymbol{z}=z_{0} \boldsymbol{e}_{0}+z_{1} \boldsymbol{e}_{1}+z_{2} \boldsymbol{e}_{2}+z_{3} \boldsymbol{e}_{3}
$$

where $\boldsymbol{e}_{0}, \boldsymbol{e}_{1}, \boldsymbol{e}_{2}, \boldsymbol{e}_{3}$ 4-orthogonal unit vectors

assume that:

$$
\boldsymbol{z}=\mathrm{d} \boldsymbol{s}=h_{0} \mathrm{~d} x_{0} \boldsymbol{e}_{0}+h_{1} \mathrm{~d} x_{1} \boldsymbol{e}_{1}+h_{2} \mathrm{~d} x_{2} \boldsymbol{e}_{2}+h_{3} \mathrm{~d} x_{3} \boldsymbol{e}_{3}
$$

And

$$
\frac{1}{\boldsymbol{z}} \equiv \frac{1}{z_{0}} \boldsymbol{e}_{0}+\frac{1}{z_{1}} \boldsymbol{e}_{1}+\frac{1}{z_{2}} \boldsymbol{e}_{2}+\frac{1}{z_{3}} \boldsymbol{e}_{3}
$$

We conclude that

$$
z_{0}=h_{0} \mathrm{~d} x_{0}, z_{1}=h_{1} \mathrm{~d} x_{1}, z_{2}=h_{2} \mathrm{~d} x_{2}, z_{3}=h_{3} \mathrm{~d} x_{3}
$$

We can write the equation of angular velocity $\vec{\omega}$ as the following

$$
\vec{\omega}=\frac{1}{2}(\nabla \times V)
$$

In our paper, we will use the last equation as the form

$$
\vec{\omega}=(\nabla \times V)
$$

where,

$\vec{\omega}: 4$-angular velocity

$\boldsymbol{V}$ : the particle 4-velocity, $\boldsymbol{V}=\frac{\mathrm{d} \boldsymbol{s}}{\mathrm{d} x_{0}}$

$\boldsymbol{\nabla}$ : bold gradient operator, $\nabla=\frac{1}{h_{0}} \frac{\partial}{\partial x_{0}} \boldsymbol{e}_{0}+\frac{1}{h_{1}} \frac{\partial}{\partial x_{1}} \boldsymbol{e}_{1}+\frac{1}{h_{2}} \frac{\partial}{\partial x_{2}} \boldsymbol{e}_{2}+\frac{1}{h_{3}} \frac{\partial}{\partial x_{3}} \boldsymbol{e}_{3}$

Now we can find the equation of 4-interval angular $d \vec{\varphi}$ as the following,

$$
\mathrm{d} \vec{\varphi}=\vec{\omega} \mathrm{d} x_{0}=(\nabla \times \boldsymbol{V}) \mathrm{d} x_{0}=\left(\nabla \times \frac{\mathrm{d} \boldsymbol{s}}{\mathrm{d} x_{0}}\right) \mathrm{d} x_{0}=\nabla \times \boldsymbol{z}
$$


The cross product of two vectors $\nabla$ and $\boldsymbol{z}$ as shown in my first paper [1] is given by

$$
\nabla \times \boldsymbol{z}=\left|\begin{array}{cccc}
\boldsymbol{e}_{0} & \boldsymbol{e}_{1} & \boldsymbol{e}_{2} & \boldsymbol{e}_{3} \\
\frac{1}{h_{0}} \frac{\partial}{\partial x_{0}} & \frac{1}{h_{1}} \frac{\partial}{\partial x_{1}} & \frac{1}{h_{2}} \frac{\partial}{\partial x_{2}} & \frac{1}{h_{3}} \frac{\partial}{\partial x_{3}} \\
z_{0} & z_{1} & z_{2} & z_{3}
\end{array}\right|
$$

then we get

$$
\begin{aligned}
\mathrm{d} \varphi_{i j} & =(\boldsymbol{\nabla} \times \boldsymbol{z})_{i j}=\left(\frac{1}{h_{i}} \frac{\partial}{\partial x_{i}} z_{j}-\frac{1}{h_{j}} \frac{\partial}{\partial x_{j}} z_{i}\right) \boldsymbol{e}_{i} \times \boldsymbol{e}_{j} \\
& =\left(\frac{\mathrm{d} x_{j}}{h_{i}} \frac{\partial}{\partial x_{i}} h_{j}-\frac{\mathrm{d} x_{i}}{h_{j}} \frac{\partial}{\partial x_{j}} h_{i}\right) \boldsymbol{v}_{i j}
\end{aligned}
$$

where

$v_{i j}$ is a complex orthogonal unit (explained in my first paper)

$\mathrm{d} x_{i, j}$ as a constant value

\subsection{The Inverse of the Interval Angular Vector $d \bar{\varphi}$}

The equation of inverse angular $\mathrm{d} \bar{\varphi}$ is defined as

$$
\mathrm{d} \overleftarrow{\varphi}=\frac{1}{z} \times \nabla
$$

Or as the following

$$
\mathrm{d} \bar{\varphi}_{i j}=\left(\frac{1}{z_{j} h_{i}} \frac{\partial}{\partial x_{i}}-\frac{1}{z_{i} h_{j}} \frac{\partial}{\partial x_{j}}\right) \boldsymbol{v}_{i j}
$$

\section{The Details of Abou Layla's Methods}

In our new way, there are two kinds of field equations $K$ and $P$, defined by

$$
\begin{gathered}
(k)=D_{\sigma \gamma} F_{\sigma \gamma} \\
(P)=\tau_{\sigma \gamma} F_{\sigma \gamma} F_{\sigma \gamma}
\end{gathered}
$$

where:

$$
\begin{gathered}
D=\eta \mathrm{d} \bar{\varphi} \\
F=\eta \mathrm{d} \vec{\varphi} \\
\tau_{\sigma \gamma}=\eta\left(\frac{1}{z_{\sigma} z_{\gamma}}\right)
\end{gathered}
$$

Or as the form,

$$
\tau_{\sigma \gamma}=\left(\begin{array}{cccc}
0 & -\frac{1}{h_{0} h_{1} \mathrm{~d} x_{0} \mathrm{~d} x_{1}} & -\frac{1}{h_{0} h_{2} \mathrm{~d} x_{0} \mathrm{~d} x_{2}} & -\frac{1}{h_{0} h_{3} \mathrm{~d} x_{0} \mathrm{~d} x_{3}} \\
\frac{1}{h_{0} h_{1} \mathrm{~d} x_{0} \mathrm{~d} x_{1}} & 0 & \frac{1}{h_{1} h_{2} \mathrm{~d} x_{1} \mathrm{~d} x_{2}} & \frac{1}{h_{1} h_{3} \mathrm{~d} x_{1} \mathrm{~d} x_{3}} \\
\frac{1}{h_{0} h_{2} \mathrm{~d} x_{0} \mathrm{~d} x_{2}} & \frac{1}{h_{1} h_{2} \mathrm{~d} x_{1} \mathrm{~d} x_{2}} & 0 & \frac{1}{h_{2} h_{3} \mathrm{~d} x_{2} \mathrm{~d} x_{3}} \\
\frac{1}{h_{0} h_{3} \mathrm{~d} x_{0} \mathrm{~d} x_{3}} & \frac{1}{h_{1} h_{3} \mathrm{~d} x_{1} \mathrm{~d} x_{3}} & \frac{1}{h_{2} h_{3} \mathrm{~d} x_{2} \mathrm{~d} x_{3}} & 0
\end{array}\right)
$$


$(k)$ kinetic field matrix in the local frame

$(P)$ potential field matrix in the local frame

$$
\eta_{a b}=\operatorname{diag}(-1,1,1,1)
$$

\subsection{Define Ricci Tensor and Ricci Scalar}

\subsubsection{Ricci Tensor}

The Ricci tensor in the local frame $\hat{R}_{\sigma \gamma}$ is equal the total field matrix as the following,

$$
\hat{R}_{\sigma \gamma}=k+P
$$

The Ricci tensor $R_{\sigma \gamma}$ in the coordinate basis can be written as

$$
R_{\sigma \gamma}=g_{\sigma \gamma} \hat{R}_{\sigma \gamma}
$$

where

$g_{\sigma \gamma}:$ The metric of the space

\subsubsection{Ricci Scalar}

The Ricci scalar $R$ is equal the sum of the elements on the main diagonal of $\hat{R}_{\sigma \gamma}$

$$
R=\operatorname{tr}\left(\hat{R}_{\sigma \gamma}\right)
$$

$\operatorname{tr}\left(\hat{R}_{\sigma \gamma}\right):$ is the trace of a matrix $\hat{R}_{\sigma \gamma}$

\subsection{Einstein Field Equation}

In general relativity, the Einstein Field Equation is defined by [2]

$$
G_{\sigma \gamma}=R_{\sigma \gamma}-\frac{1}{2} R g_{\sigma \gamma}
$$

And the equation of stress-energy tensor $T_{\sigma \gamma}$ is

$$
G_{\sigma \gamma}=\kappa T_{\sigma \gamma}
$$

where:

$\kappa:$ is a constant, $\kappa=\frac{8 \pi G}{c^{4}}$

İn our paper we suppose that

$$
\hat{G}_{\sigma \gamma}=\hat{R}_{\sigma \gamma}-\frac{1}{2} R I
$$

The Einstein Field Equation $G$ in the coordinate basis can be written as

$$
G=g_{\sigma \gamma} \hat{G}
$$

\subsection{Important Note}

\subsubsection{Options of the Potential Matrix}

To find the potential matrix, there are two possibility option

Option 1

$$
\begin{gathered}
(P)=\tau_{\sigma \gamma} F_{\sigma \gamma} F_{\sigma \gamma}=\left(\tau_{\sigma \gamma} F_{\sigma \gamma}\right) F_{\sigma \gamma}=S F_{\sigma \gamma} \\
\vec{S}=\tau_{\sigma \gamma} F_{\sigma \gamma}
\end{gathered}
$$


Option 2

$$
(P)=\tau_{\sigma \gamma} F_{\sigma \gamma} F_{\sigma \gamma}=\tau_{\sigma \gamma}\left(F_{\sigma \gamma} F_{\sigma \gamma}\right)=\tau_{\sigma \gamma} F_{\sigma \gamma}^{2}
$$

\subsubsection{The Easiest Way to Find the Components of Potential Matrix} We can easily find the components of potential matrix by Assuming that,

$$
\delta_{i j}=\frac{\mathrm{d} x_{i}}{\mathrm{~d} x_{j}}
$$

Therefore

$$
\begin{aligned}
\delta_{i j}=1 \text { if } i=j, \quad \delta_{i j}=0 \quad \text { if } i \neq j \\
\delta_{i j} \delta_{k l}=\frac{\mathrm{d} x_{i}}{\mathrm{~d} x_{j}} \frac{\mathrm{d} x_{k}}{\mathrm{~d} x_{l}}=\frac{1}{\mathrm{~d} x_{j}} \frac{1}{\mathrm{~d} x_{l}} \mathrm{~d} x_{i} \mathrm{~d} x_{k}
\end{aligned}
$$

$\delta_{i j} \delta_{i k}=\frac{\mathrm{d} x_{i}}{\mathrm{~d} x_{j}} \frac{\mathrm{d} x_{i}}{\mathrm{~d} x_{k}}=\frac{1}{\mathrm{~d} x_{j}} \frac{1}{\mathrm{~d} x_{k}} \mathrm{~d} x_{i} \mathrm{~d} x_{i}=0$, it looks like $\mathrm{d} x_{i} \mathrm{~d} x_{i} \equiv 0$ because $k, j$ always different $k \neq j$

For $i \neq j \neq k, \mathrm{~d} x_{l} \neq 0$ we find

$$
\delta_{i j} \delta_{l k}=\frac{\mathrm{d} x_{i}}{\mathrm{~d} x_{j}} \frac{\mathrm{d} x_{l}}{\mathrm{~d} x_{k}}=\left(\frac{1}{\mathrm{~d} x_{j} \mathrm{~d} x_{k}} \mathrm{~d} x_{i}\right) \mathrm{d} x_{l}=0, \text { it looks like } \frac{1}{\mathrm{~d} x_{j} \mathrm{~d} x_{k}} \mathrm{~d} x_{i} \equiv 0
$$

\section{Discussion}

In this discussion, we will calculate the components of the Ricci tensor, Ricci scalar and Einstein field equation for many metrics directly by using our new way, we will see that all results will be correct and consistent with the calculations founded in General Relativity Books

\subsection{Calculate the Components of the Ricci Tensor}

\section{EXAMPLE 1}

Consider the metric

$$
\mathrm{d} s^{2}=-\mathrm{d} t^{2}+\mathrm{e}^{2 b}(t, r) \mathrm{d} r^{2}+R^{2}(t, r) \mathrm{d} \phi^{2}
$$

and use Abou Layla's structure equations to find the components of Ricci tensor in the local frame

\section{SOLUTION}

Looking at the metric, we define the orthonormal vector $\boldsymbol{z}$ as the following

$$
\boldsymbol{z}=\mathrm{d} t \boldsymbol{L}+\mathrm{e}^{b}(t, r) \mathrm{d} r \boldsymbol{i}+0 \times h_{\theta} \mathrm{d} \theta \boldsymbol{j}+R(t, r) \mathrm{d} \phi \boldsymbol{k}
$$

Then we have

$$
\nabla=\frac{\partial}{\partial t} \boldsymbol{L}+\frac{1}{\mathrm{e}^{b}} \frac{\partial}{\partial r} \boldsymbol{i}+0 \frac{\partial}{\partial \theta} \boldsymbol{j}+\frac{1}{R} \frac{\partial}{\partial \phi} \boldsymbol{k}
$$

The equation of 4-interval angular $\mathrm{d} \vec{\varphi}$ is given by

$$
\nabla \times \boldsymbol{z}=\left|\begin{array}{cccc}
L & i & j & k \\
\frac{\partial}{\partial t} & \frac{1}{\mathrm{e}^{b}} \frac{\partial}{\partial r} & 0 \frac{\partial}{\partial \theta} & \frac{1}{R} \frac{\partial}{\partial \phi} \\
1 \mathrm{~d} t & \mathrm{e}^{b} \mathrm{~d} r & 0 \mathrm{~d} \theta & R \mathrm{~d} \phi
\end{array}\right|,\left(\mathrm{d} x_{i} \text { as a constant value }\right)
$$


then we get

$$
\begin{aligned}
\mathrm{d} \vec{\varphi}= & \nabla \times \boldsymbol{z} \\
= & \left(\frac{\partial}{\partial t} \mathrm{e}^{b} \mathrm{~d} r-\frac{1}{\mathrm{e}^{b}} \frac{\partial}{\partial r} \mathrm{~d} t\right) L \times i+\left(\frac{\partial}{\partial t} 0 \mathrm{~d} \theta-0 \frac{\partial}{\partial \theta} 1 \mathrm{~d} t\right) L \times j \\
& +\left(\frac{\partial}{\partial t} R \mathrm{~d} \phi-\frac{1}{R} \frac{\partial}{\partial \phi} 1 \mathrm{~d} t\right) L \times k+\left(\frac{1}{\mathrm{e}^{b}} \frac{\partial}{\partial r} 0 \mathrm{~d} \theta-0 \frac{\partial}{\partial \theta} \mathrm{e}^{b} \mathrm{~d} r\right) i \times j \\
& +\left(\frac{1}{\mathrm{e}^{b}} \frac{\partial}{\partial r} R \mathrm{~d} \phi-\frac{1}{R} \frac{\partial}{\partial \phi} \mathrm{e}^{b} \mathrm{~d} r\right) i \times k+\left(0 \frac{\partial}{\partial \theta} R \mathrm{~d} \phi-\frac{1}{R} \frac{\partial}{\partial \phi} 0 \mathrm{~d} \theta\right) j \times k \\
& \mathrm{~d} \vec{\varphi}=\left(\frac{\partial}{\partial t} \mathrm{e}^{b} \mathrm{~d} r\right) v^{01}+(0) v^{02}+\left(\frac{\partial}{\partial t} R \mathrm{~d} \phi\right) v^{03}+(0) v^{12} \\
& +\left(\mathrm{e}^{-b} \frac{\partial}{\partial r} R \mathrm{~d} \phi\right) v^{13}+(0) v^{23}
\end{aligned}
$$

Therefore, we have

$$
\begin{aligned}
\mathrm{d} \varphi_{\sigma \gamma} & =\left(\begin{array}{cccc}
0 & \frac{\partial}{\partial t}\left(\mathrm{e}^{b} \mathrm{~d} r\right) & 0 & \frac{\partial}{\partial t}(R \mathrm{~d} \phi) \\
-\frac{\partial}{\partial t}\left(\mathrm{e}^{b} \mathrm{~d} r\right) & 0 & 0 & \mathrm{e}^{-b} \frac{\partial}{\partial r}(R \mathrm{~d} \phi) \\
0 & 0 & 0 & 0 \\
-\frac{\partial}{\partial t}(R \mathrm{~d} \phi) & -\mathrm{e}^{-b} \frac{\partial}{\partial r}(R \mathrm{~d} \phi) & 0 & 0
\end{array}\right) \\
& =\left(\begin{array}{cccc}
0 & \frac{\partial b}{\partial t} \mathrm{e}^{b} \mathrm{~d} r & 0 & \frac{\partial R}{\partial t} \mathrm{~d} \phi \\
-\frac{\partial b}{\partial t} \mathrm{e}^{b} \mathrm{~d} r & 0 & 0 & \mathrm{e}^{-b} \frac{\partial R}{\partial r} \mathrm{~d} \phi \\
0 & 0 & 0 & 0 \\
-\frac{\partial R}{\partial t} \mathrm{~d} \phi & -\mathrm{e}^{-b} \frac{\partial R}{\partial r} \mathrm{~d} \phi & 0 & 0
\end{array}\right)
\end{aligned}
$$

Thus,

$$
F_{\sigma \gamma}=\eta \mathrm{d} \varphi_{\sigma \gamma}=\left(\begin{array}{cccc}
0 & -\frac{\partial b}{\partial t} \mathrm{e}^{b} \mathrm{~d} r & 0 & -\frac{\partial R}{\partial t} \mathrm{~d} \phi \\
-\frac{\partial b}{\partial t} \mathrm{e}^{b} \mathrm{~d} r & 0 & 0 & \mathrm{e}^{-b} \frac{\partial R}{\partial r} \mathrm{~d} \phi \\
0 & 0 & 0 & 0 \\
-\frac{\partial R}{\partial t} \mathrm{~d} \phi & -\mathrm{e}^{-b} \frac{\partial R}{\partial r} \mathrm{~d} \phi & 0 & 0
\end{array}\right)
$$

We can find the components of matrix $\mathrm{d} \bar{\varphi}_{\sigma \gamma}$ directly from matrix $\mathrm{d} \vec{\varphi}_{\sigma \gamma}$ as the following

$$
\mathrm{d} \bar{\varphi}_{\sigma \gamma}=\left(\begin{array}{cccc}
0 & \frac{1}{\left(\mathrm{e}^{b} \mathrm{~d} r\right)} \frac{\partial}{\partial t} & 0 & \frac{1}{(R \mathrm{~d} \phi)} \frac{\partial}{\partial t} \\
-\frac{1}{\left(\mathrm{e}^{b} \mathrm{~d} r\right)} \frac{\partial}{\partial t} & 0 & 0 & \frac{\mathrm{e}^{-b}}{(R \mathrm{~d} \phi)} \frac{\partial}{\partial r} \\
0 & 0 & 0 & 0 \\
-\frac{1}{(R \mathrm{~d} \phi)} \frac{\partial}{\partial t} & -\frac{\mathrm{e}^{-b}}{(R \mathrm{~d} \phi)} \frac{\partial}{\partial r} & 0 & 0
\end{array}\right)
$$


Then,

$$
D_{\sigma \gamma}=\eta \mathrm{d} \bar{\varphi}_{\sigma \gamma}=\left(\begin{array}{cccc}
0 & -\frac{1}{\mathrm{e}^{b} \mathrm{~d} r} \frac{\partial}{\partial t} & 0 & -\frac{1}{R \mathrm{~d} \phi} \frac{\partial}{\partial t} \\
-\frac{1}{\mathrm{e}^{b} \mathrm{~d} r} \frac{\partial}{\partial t} & 0 & 0 & \frac{\mathrm{e}^{-b}}{R \mathrm{~d} \phi} \frac{\partial}{\partial r} \\
0 & 0 & 0 & 0 \\
-\frac{1}{R \mathrm{~d} \phi} \frac{\partial}{\partial t} & -\frac{e^{-b}}{R \mathrm{~d} \phi} \frac{\partial}{\partial r} & 0 & 0
\end{array}\right)
$$

We now proceed to find the Ricci tensor $\hat{R}_{\sigma \gamma}$, there are three steps: Step1: The components of the kinetic matrix

$$
\begin{aligned}
(k)=D_{\sigma \gamma} F_{\sigma \gamma}= & \left(\begin{array}{ccccc}
0 & -\frac{1}{\mathrm{e}^{b} \mathrm{~d} r} \frac{\partial}{\partial t} & 0 & -\frac{1}{R \mathrm{~d} \phi} \frac{\partial}{\partial t} \\
-\frac{1}{\mathrm{e}^{b} \mathrm{~d} r} \frac{\partial}{\partial t} & 0 & 0 & \frac{\mathrm{e}^{-b}}{R \mathrm{~d} \phi} \frac{\partial}{\partial r} \\
0 & 0 & 0 & 0 \\
-\frac{1}{R \mathrm{~d} \phi} \frac{\partial}{\partial t} & -\frac{\mathrm{e}^{-b}}{R \mathrm{~d} \phi} \frac{\partial}{\partial r} & 0 & 0
\end{array}\right) \\
& \times\left(\begin{array}{ccccc}
0 & -\frac{\partial b}{\partial t} \mathrm{e}^{b} \mathrm{~d} r & 0 & -\frac{\partial R}{\partial t} \mathrm{~d} \phi \\
-\frac{\partial b}{\partial t} \mathrm{e}^{b} \mathrm{~d} r & 0 & 0 & \mathrm{e}^{-b} \frac{\partial R}{\partial r} \mathrm{~d} \phi \\
0 & 0 & 0 & 0 \\
-\frac{\partial R}{\partial t} \mathrm{~d} \phi & -\mathrm{e}^{-b} \frac{\partial R}{\partial r} \mathrm{~d} \phi & 0 & 0
\end{array}\right)
\end{aligned}
$$

The nonzero components of kinetic matrix $k_{\sigma \gamma}$ are

$$
\begin{gathered}
k_{t t}=\frac{1}{\mathrm{e}^{b} \mathrm{~d} r} \frac{\partial}{\partial t}\left(\frac{\partial b}{\partial t} \mathrm{e}^{b} \mathrm{~d} r\right)+\frac{1}{R \mathrm{~d} \phi} \frac{\partial}{\partial t}\left(\frac{\partial R}{\partial t} \mathrm{~d} \phi\right) \\
k_{t t}=\frac{1}{\mathrm{e}^{b}} \frac{\partial^{2} b}{\partial t^{2}} \mathrm{e}^{b}+\frac{1}{\mathrm{e}^{b}}\left(\frac{\partial b}{\partial t}\right)^{2} \mathrm{e}^{b}+\frac{1}{R} \frac{\partial^{2} R}{\partial t^{2}}=\frac{\partial^{2} b}{\partial t^{2}}+\left(\frac{\partial b}{\partial t}\right)^{2}+\frac{1}{R} \frac{\partial^{2} R}{\partial t^{2}} \\
k_{t r}=\frac{1}{R \mathrm{~d} \phi} \frac{\partial}{\partial t}\left(\mathrm{e}^{-b} \frac{\partial R}{\partial r} \mathrm{~d} \phi\right)=-\frac{\mathrm{e}^{-b}}{R} \frac{\partial b}{\partial t} \frac{\partial R}{\partial r}+\frac{\mathrm{e}^{-b}}{R} \frac{\partial^{2} R}{\partial t \partial r} \\
k_{r r}=\frac{1}{\mathrm{e}^{b} \mathrm{~d} r} \frac{\partial}{\partial t}\left(\frac{\partial b}{\partial t} \mathrm{e}^{b} \mathrm{~d} r\right)-\frac{\mathrm{e}^{-b}}{R \mathrm{~d} \phi} \frac{\partial}{\partial r}\left(\mathrm{e}^{-b} \frac{\partial R}{\partial r} \mathrm{~d} \phi\right) \\
k_{r r}=\frac{\partial^{2} b}{\partial t^{2}}+\left(\frac{\partial b}{\partial t}\right)^{2}+\frac{\mathrm{e}^{-2 b}}{R} \frac{\partial b}{\partial r} \frac{\partial R}{\partial r}-\frac{\mathrm{e}^{-2 b}}{R} \frac{\partial^{2} R}{\partial r^{2}} \\
k_{\phi \phi}=\frac{1}{R \mathrm{~d} \phi} \frac{\partial}{\partial t}\left(\frac{\partial R}{\partial t} \mathrm{~d} \phi\right)-\frac{\mathrm{e}^{-b}}{R \mathrm{~d} \phi} \frac{\partial}{\partial r}\left(\mathrm{e}^{-b} \frac{\partial R}{\partial r} \mathrm{~d} \phi\right) \\
k_{\phi \phi}=\frac{1}{R} \frac{\partial^{2} R}{\partial t^{2}}+\frac{\mathrm{e}^{-2 b}}{R} \frac{\partial b}{\partial r} \frac{\partial R}{\partial r}-\frac{\mathrm{e}^{-2 b}}{R} \frac{\partial^{2} R}{\partial r^{2}}
\end{gathered}
$$

The next step is to find the components of the potential filed matrix:

Using option 2 as shown in (3.12), we can calculate the square of vector $\vec{F}$ 
form as,

$$
F_{\sigma \gamma}^{2}=F_{\sigma \gamma} F_{\sigma \gamma}=\left(\begin{array}{cccc}
0 & 0 & 0 & -\frac{\partial b}{\partial t} \mathrm{e}^{b} \mathrm{~d} r \frac{\partial R}{\partial r} \mathrm{e}^{-b} \mathrm{~d} \phi \\
0 & 0 & 0 & \frac{\partial b}{\partial t} \mathrm{e}^{b} \mathrm{~d} r \frac{\partial R}{\partial t} \mathrm{~d} \phi \\
0 & 0 & 0 & 0 \\
\frac{\partial R}{\partial r} \mathrm{e}^{-b} \mathrm{~d} \phi \frac{\partial b}{\partial t} \mathrm{e}^{b} \mathrm{~d} r & \frac{\partial R}{\partial t} \mathrm{~d} \phi \frac{\partial b}{\partial t} \mathrm{e}^{b} \mathrm{~d} r & 0 & 0
\end{array}\right)
$$

Then we obtain

$$
\begin{aligned}
P_{\sigma \gamma}= & \tau_{\sigma \gamma} F_{\sigma \gamma}^{2} \\
= & \left(\begin{array}{cccc}
0 & -\frac{1}{\mathrm{e}^{b} \mathrm{~d} t \mathrm{~d} r} & 0 & -\frac{1}{R \mathrm{~d} t \mathrm{~d} \phi} \\
\frac{1}{\mathrm{e}^{b} \mathrm{~d} t \mathrm{~d} r} & 0 & 0 & \frac{1}{\mathrm{e}^{b} R \mathrm{~d} r \mathrm{~d} \phi} \\
0 & 0 & 0 & 0 \\
\frac{1}{R \mathrm{~d} t \mathrm{~d} \phi} & \frac{1}{\mathrm{e}^{b} R \mathrm{~d} r \mathrm{~d} \phi} & 0 & 0
\end{array}\right) \\
& \times\left(\begin{array}{ccccc}
0 & 0 & -\frac{\partial b}{\partial t} \mathrm{e}^{b} \mathrm{~d} r \frac{\partial R}{\partial r} \mathrm{e}^{-b} \mathrm{~d} \phi \\
0 & & 0 & \frac{\partial b}{\partial t} \mathrm{e}^{b} \mathrm{~d} r \frac{\partial R}{\partial t} \mathrm{~d} \phi \\
\frac{\partial R}{\partial r} \mathrm{e}^{-b} \mathrm{~d} \phi \frac{\partial b}{\partial t} \mathrm{e}^{b} \mathrm{~d} r & \frac{\partial R}{\partial t} \mathrm{~d} \phi \frac{\partial b}{\partial t} \mathrm{e}^{b} \mathrm{~d} r & 0 & 0
\end{array}\right)
\end{aligned}
$$

And the matrix $P_{\sigma \gamma}$ is

$$
P_{\sigma \gamma}=\left(\begin{array}{cccc}
0 & 0 & 0 & 0 \\
0 & \frac{1}{R} \frac{\partial R}{\partial t} \frac{\partial b}{\partial t} & 0 & 0 \\
0 & 0 & 0 & 0 \\
0 & 0 & 0 & \frac{1}{R} \frac{\partial b}{\partial t} \frac{\partial R}{\partial t}
\end{array}\right)
$$

\section{Step3: Summation kinetic matrix and potential matrix}

By summation $(K)$ and $(P)$ we get Ricci tensor $\hat{R}_{\sigma \gamma}$ as shown in (3.6) Thus

$$
\begin{gathered}
\hat{R}_{t t}=\frac{\partial^{2} b}{\partial t^{2}}+\left(\frac{\partial b}{\partial t}\right)^{2}+\frac{1}{R} \frac{\partial^{2} R}{\partial t^{2}} \\
\hat{R}_{t r}=-\frac{\mathrm{e}^{-b}}{R} \frac{\partial b}{\partial t} \frac{\partial R}{\partial r}+\frac{\mathrm{e}^{-b}}{R} \frac{\partial^{2} R}{\partial t \partial r} \\
\hat{R}_{r r}=\frac{\partial^{2} b}{\partial t^{2}}+\left(\frac{\partial b}{\partial t}\right)^{2}+\frac{\mathrm{e}^{-2 b}}{R} \frac{\partial b}{\partial r} \frac{\partial R}{\partial r}-\frac{\mathrm{e}^{-2 b}}{R} \frac{\partial^{2} R}{\partial r^{2}}+\frac{1}{R} \frac{\partial R}{\partial t} \frac{\partial b}{\partial t} \\
\hat{R}_{\phi \phi}=\frac{1}{R} \frac{\partial^{2} R}{\partial t^{2}}+\frac{\mathrm{e}^{-2 b}}{R} \frac{\partial b}{\partial r} \frac{\partial R}{\partial r}-\frac{\mathrm{e}^{-2 b}}{R} \frac{\partial^{2} R}{\partial r^{2}}+\frac{1}{R} \frac{\partial R}{\partial t} \frac{\partial b}{\partial t}
\end{gathered}
$$




\subsection{Calculate the Components of the Stress-Energy Tensor $T$ \\ EXAMPLE 2}

Using the results of Example above, find the components of the stress-energy tensor $T$

\section{SOLUTION}

First, we need to find the Ricci scalar $R$

$$
\begin{aligned}
& R= \operatorname{tr}\left(\hat{R}_{\sigma \gamma}\right)=\left[\frac{\partial^{2} b}{\partial t^{2}}+\left(\frac{\partial b}{\partial t}\right)^{2}+\frac{1}{R} \frac{\partial^{2} R}{\partial t^{2}}\right] \\
&+\left[\frac{\partial^{2} b}{\partial t^{2}}+\left(\frac{\partial b}{\partial t}\right)^{2}-\frac{\mathrm{e}^{-2 b}}{R} \frac{\partial^{2} R}{\partial r^{2}}+\frac{\mathrm{e}^{-2 b}}{R} \frac{\partial b}{\partial r} \frac{\partial R}{\partial r}+\frac{1}{R} \frac{\partial R}{\partial t} \frac{\partial b}{\partial t}\right] \\
&+\left[\frac{1}{R} \frac{\partial^{2} R}{\partial t^{2}}-\frac{\mathrm{e}^{-2 b}}{R} \frac{\partial^{2} R}{\partial r^{2}}+\frac{\mathrm{e}^{-2 b}}{R} \frac{\partial b}{\partial r} \frac{\partial R}{\partial r}+\frac{1}{R} \frac{\partial R}{\partial t} \frac{\partial b}{\partial t}\right] \\
& R=2\left[\frac{\partial^{2} b}{\partial t^{2}}+\left(\frac{\partial b}{\partial t}\right)^{2}+\frac{1}{R} \frac{\partial^{2} R}{\partial t^{2}}-\frac{\mathrm{e}^{-2 b}}{R} \frac{\partial^{2} R}{\partial r^{2}}+\frac{\mathrm{e}^{-2 b}}{R} \frac{\partial b}{\partial r} \frac{\partial R}{\partial r}+\frac{1}{R} \frac{\partial R}{\partial t} \frac{\partial b}{\partial t}\right]
\end{aligned}
$$

Using (3.9), we can find the components of Einstein's tensor as the following

$$
\begin{gathered}
\hat{G}_{t t}=\frac{\mathrm{e}^{-2 b}}{R} \frac{\partial^{2} R}{\partial r^{2}}-\frac{\mathrm{e}^{-2 b}}{R} \frac{\partial b}{\partial r} \frac{\partial R}{\partial r}-\frac{1}{R} \frac{\partial b}{\partial t} \frac{\partial R}{\partial t} \\
\hat{G}_{t r}=\frac{\mathrm{e}^{-b}}{R} \frac{\partial^{2} R}{\partial t \partial r}-\frac{\mathrm{e}^{-b}}{R} \frac{\partial b}{\partial t} \frac{\partial R}{\partial r} \\
\hat{G}_{r r}=-\frac{1}{R} \frac{\partial^{2} R}{\partial t^{2}} \\
\hat{G}_{r r}=-\left[\frac{\partial^{2} b}{\partial t^{2}}+\left(\frac{\partial b}{\partial t}\right)^{2}\right]
\end{gathered}
$$

Now, using transformation

$$
G=g_{\sigma \gamma} \hat{G}
$$

we obtain

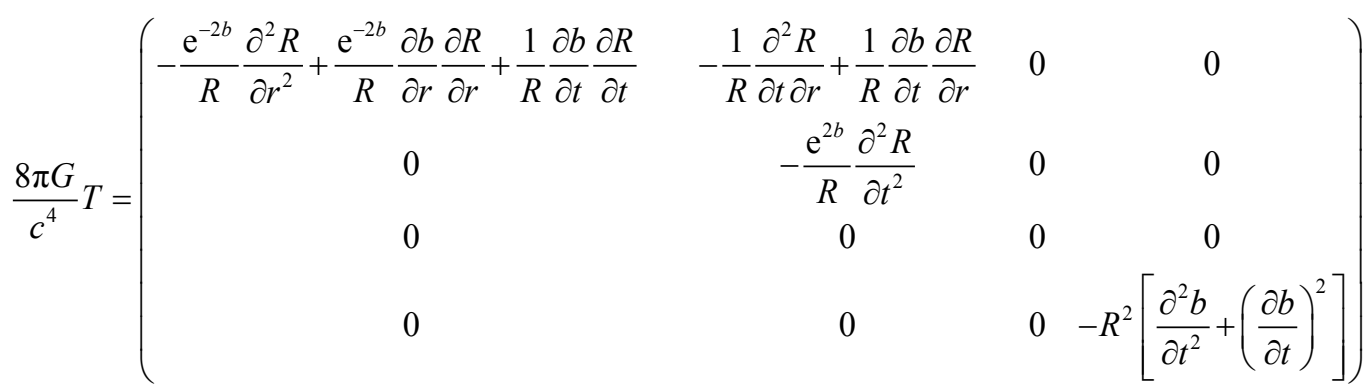

\subsection{Solution of the Robertson-Walker Metric}

EXAMPLE 3

Consider the Robertson-Walker metric

$$
\mathrm{d} \boldsymbol{s}^{2}=-\mathrm{d} t^{2}+\frac{a^{2}}{1-k r^{2}} \mathrm{~d} r^{2}+a^{2}(t) r^{2} \mathrm{~d} \theta^{2}+a^{2}(t) r^{2} \sin ^{2} \theta \mathrm{d} \phi^{2}
$$


and use Abou Layla's structure equations to find the components of the Ricci tensor and the Ricci scalar

\section{SOLUTION}

For the given metric, we see that we can define the orthonormal vector $\boldsymbol{Z}$ as the following

$$
\boldsymbol{z}=-\mathrm{d} t \boldsymbol{L}+\frac{a}{\sqrt{1-k r^{2}}} \mathrm{~d} r \boldsymbol{i}+a(t) r \mathrm{~d} \theta \boldsymbol{j}+a(t) r \sin \theta \mathrm{d} \phi \boldsymbol{k}
$$

Then we have

$$
\boldsymbol{\nabla}=\frac{\partial}{\partial t} \boldsymbol{L}+\frac{\sqrt{1-k r^{2}}}{a} \frac{\partial}{\partial r} \boldsymbol{i}+\frac{1}{a r} \frac{\partial}{\partial \theta} \boldsymbol{j}+\frac{1}{a r \sin \theta} \frac{\partial}{\partial \phi} \boldsymbol{k}
$$

Now we calculate the equation of 4-interval angular $\mathrm{d} \vec{\varphi}$

$$
\nabla \times \boldsymbol{z}=\left|\begin{array}{cccc}
L & i & j & k \\
\frac{\partial}{\partial t} & \frac{\sqrt{1-k r^{2}}}{a} \frac{\partial}{\partial r} & \frac{1}{\operatorname{ar}} \frac{\partial}{\partial \theta} & \frac{1}{\operatorname{arsin} \theta} \frac{\partial}{\partial \phi} \\
\mathrm{d} t & \frac{a}{\sqrt{1-k r^{2}}} \mathrm{~d} r & \operatorname{ard} \theta & \operatorname{ar} \sin \theta \mathrm{d} \phi
\end{array}\right|,\left(\mathrm{d} x_{i} \text { as a constant value }\right)
$$

then we get

$$
\left.\begin{array}{c}
\mathrm{d} \vec{\varphi}=\nabla \times \boldsymbol{z} \\
=\left(\frac{\partial}{\partial t} \frac{a}{\sqrt{1-k r^{2}}} \mathrm{~d} r-\frac{\sqrt{1-k r^{2}}}{a} \frac{\partial}{\partial r} \mathrm{~d} t\right) L \times i \\
+\left(\frac{\partial}{\partial t} \operatorname{ard} \theta-\frac{1}{a r} \frac{\partial}{\partial \theta} \mathrm{d} t\right) L \times j \\
+\left(\frac{\partial}{\partial t} \operatorname{ar} \sin \theta \mathrm{d} \phi-\frac{1}{a r \sin \theta} \frac{\partial}{\partial \phi} \mathrm{d} t\right) L \times k \\
+\left(\frac{\sqrt{1-k r^{2}}}{a} \frac{\partial}{\partial r} \operatorname{ard} \theta-\frac{1}{a r} \frac{\partial}{\partial \theta} \frac{a}{\sqrt{1-k r^{2}}} \mathrm{~d} r\right) i \times j \\
+\left(\frac{\sqrt{1-k r^{2}}}{a} \frac{\partial}{\partial r} \operatorname{arsin} \theta \mathrm{d} \phi-\frac{1}{a r \sin \theta} \frac{\partial}{\partial \phi} \frac{a}{\sqrt{1-k r^{2}}} \mathrm{~d} r\right) i \times k
\end{array}\right) j
$$


We can find the components of matrix $\mathrm{d} \bar{\varphi}_{\sigma \gamma}$ directly from matrix $\mathrm{d} \vec{\varphi}_{\sigma \gamma}$ as the following

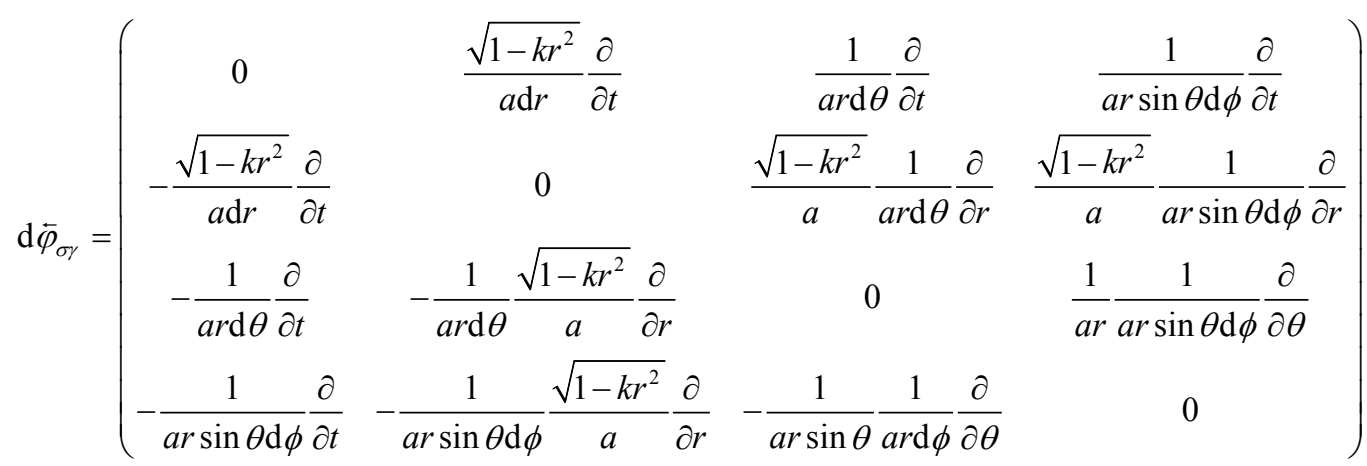

We now proceed to find the Ricci tensor $\hat{R}_{\sigma \gamma}$

Step1: The components of the kinetic matrix

$$
\begin{aligned}
& D_{\sigma \gamma}=\eta \mathrm{d} \bar{\varphi}_{\sigma \gamma}=\left(\begin{array}{cccc}
0 & -\frac{\sqrt{1-k r^{2}}}{a \mathrm{~d} r} \frac{\partial}{\partial t} & -\frac{1}{a r \mathrm{~d} \theta} \frac{\partial}{\partial t} & -\frac{1}{a r \sin \theta \mathrm{d} \phi} \frac{\partial}{\partial t} \\
-\frac{\sqrt{1-k r^{2}}}{a \mathrm{~d} r} \frac{\partial}{\partial t} & 0 & \frac{\sqrt{1-k r^{2}}}{a^{2} r \mathrm{~d} \theta} \frac{\partial}{\partial r} & \frac{\sqrt{1-k r^{2}}}{a^{2} r \sin \theta \mathrm{d} \phi} \frac{\partial}{\partial r} \\
-\frac{1}{a r \mathrm{~d} \theta} \frac{\partial}{\partial t} & -\frac{\sqrt{1-k r^{2}}}{a^{2} r \mathrm{~d} \theta} \frac{\partial}{\partial r} & 0 & 1 \\
-\frac{1}{a r \sin \theta \mathrm{d} \phi} \frac{\partial}{\partial t} & -\frac{\sqrt{1-k r^{2}}}{a^{2} r \sin \theta \mathrm{d} \phi} \frac{\partial}{\partial r} & -\frac{1}{a^{2} r^{2} \sin \theta \mathrm{d} \phi} \frac{\partial}{\partial \theta} & 0
\end{array}\right) \\
& F_{\sigma \gamma}=\eta \mathrm{d} \vec{\varphi}_{\sigma \gamma}=\left(\begin{array}{cccc}
0 & -\frac{\dot{a}}{\sqrt{1-k r^{2}}} \mathrm{~d} r & -\dot{a} r \mathrm{~d} \theta & -r \dot{a} \sin \theta \mathrm{d} \phi \\
-\frac{\dot{a}}{\sqrt{1-k r^{2}}} \mathrm{~d} r & 0 & \sqrt{1-k r^{2}} \mathrm{~d} \theta & \sin \theta \sqrt{1-k r^{2}} \mathrm{~d} \phi \\
-r \dot{a} \mathrm{~d} \theta & -\sqrt{1-k r^{2}} \mathrm{~d} \theta & 0 & \cos \theta \mathrm{d} \phi \\
-r \dot{a} \sin \theta \mathrm{d} \phi & -\sin \theta \sqrt{1-k r^{2}} \mathrm{~d} \phi & -\cos \theta \mathrm{d} \phi & 0
\end{array}\right)
\end{aligned}
$$

By product of two matrix $D_{\sigma \gamma}$ and $F_{\sigma \gamma}$ we can find the components of the kinetic field matrix $k_{\sigma \gamma}$ as the following

$$
\begin{gathered}
k_{00}=\frac{\sqrt{1-k r^{2}}}{a \mathrm{~d} r} \frac{\partial}{\partial t}\left(\frac{\dot{a}}{\sqrt{1-k r^{2}}}\right)+\frac{1}{a r \mathrm{~d} \theta} \frac{\partial}{\partial t}(r \dot{a} \mathrm{~d} \theta)+\frac{1}{a r \sin \theta \mathrm{d} \phi} \frac{\partial}{\partial t}(r \dot{a} \sin \theta \mathrm{d} \phi) \\
k_{00}=\frac{\ddot{a}}{a}+\frac{\ddot{a}}{a}+\frac{\ddot{a}}{a}=3 \frac{\ddot{a}}{a} \\
k_{11}=\frac{\sqrt{1-k r^{2}}}{a d r} \frac{\partial}{\partial t}\left(\frac{\dot{a}}{\sqrt{1-k r^{2}}} \mathrm{~d} r\right)+\frac{\sqrt{1-k r^{2}}}{a^{2} r \mathrm{~d} \theta} \frac{\partial}{\partial r}\left(-\sqrt{1-k r^{2}} \mathrm{~d} \theta\right) \\
+\frac{\sqrt{1-k r^{2}}}{a^{2} r \sin \theta \mathrm{d} \phi} \frac{\partial}{\partial r}\left(-\sin \theta \sqrt{1-k r^{2}} \mathrm{~d} \phi\right) \\
k_{11}=\frac{\ddot{a}}{a}+\frac{k}{a^{2}}+\frac{k}{a^{2}}=\frac{a \ddot{a}+2 k}{a^{2}}
\end{gathered}
$$




$$
\begin{gathered}
k_{22}=\frac{1}{a r \mathrm{~d} \theta} \frac{\partial}{\partial t}(\dot{a} r \mathrm{~d} \theta)-\frac{\sqrt{1-k r^{2}}}{a^{2} r \mathrm{~d} \theta} \frac{\partial}{\partial r}\left(\sqrt{1-k r^{2}} \mathrm{~d} \theta\right) \\
+\frac{1}{a^{2} r^{2} \sin \theta \mathrm{d} \phi} \frac{\partial}{\partial \theta}(-\cos \theta \mathrm{d} \phi) \\
k_{22}=\frac{\ddot{a}}{a}+\frac{k}{a^{2}}+\frac{1}{a^{2} r^{2}} \\
k_{33}=\frac{1}{a r \sin \theta \mathrm{d} \phi} \frac{\partial}{\partial t}(r \dot{a} \sin \theta \mathrm{d} \phi)-\frac{\sqrt{1-k r^{2}}}{a^{2} r \sin \theta \mathrm{d} \phi} \frac{\partial}{\partial r}\left(\sin \theta \sqrt{1-k r^{2}} \mathrm{~d} \phi\right) \\
-\frac{1}{a^{2} r^{2} \sin \theta \mathrm{d} \phi} \frac{\partial}{\partial \theta}(\cos \theta \mathrm{d} \phi) \\
k_{33}=\frac{\ddot{a}}{a}+\frac{k}{a^{2}}+\frac{1}{a^{2} r^{2}}
\end{gathered}
$$

Step 2: The components of the potential filed matrix:

By using (3.5) we can find matrix $(\tau)$ as follows

$$
\tau_{\sigma \gamma}=\left(\begin{array}{cccc}
0 & -\frac{\sqrt{1-k r^{2}}}{a \mathrm{~d} t \mathrm{~d} r} & -\frac{1}{a r \mathrm{~d} t \mathrm{~d} \theta} & -\frac{1}{a r \sin \theta \mathrm{d} t \mathrm{~d} \phi} \\
\frac{\sqrt{1-k r^{2}}}{a \mathrm{~d} r \mathrm{~d} t} & 0 & \frac{\sqrt{1-k r^{2}}}{r a^{2} \mathrm{~d} r \mathrm{~d} \theta} & \frac{\sqrt{1-k r^{2}}}{a^{2} r \sin \theta \mathrm{d} r \mathrm{~d} \phi} \\
\frac{1}{a r \mathrm{~d} \theta \mathrm{d} t} & \frac{\sqrt{1-k r^{2}}}{a^{2} r \mathrm{~d} \theta \mathrm{d} r} & 0 & \frac{1}{a^{2} r^{2} \sin \theta \mathrm{d} \theta \mathrm{d} \phi} \\
\frac{1}{a r \sin \theta \mathrm{d} \phi \mathrm{d} t} & \frac{\sqrt{1-k r^{2}}}{a^{2} r \sin \theta \mathrm{d} \phi \mathrm{d} r} & \frac{1}{a^{2} r^{2} \sin \theta \mathrm{d} \phi \mathrm{d} \theta} & 0
\end{array}\right)
$$

Using option 1 as shown in (3.10) and (3.11), we can calculate the product of two matrix $\tau_{\sigma \gamma}$ and $\mathrm{d} \varphi_{\sigma \gamma}$ as follows,

$$
\begin{gathered}
\left(\begin{array}{cccc}
0 & -\frac{\sqrt{1-k r^{2}}}{a \mathrm{~d} t \mathrm{~d} r} & -\frac{1}{a r \mathrm{~d} t \mathrm{~d} \theta} & -\frac{1}{a r \sin \theta \mathrm{d} t \mathrm{~d} \phi} \\
\frac{\sqrt{1-k r^{2}}}{a \mathrm{~d} r \mathrm{~d} t} & 0 & \frac{\sqrt{1-k r^{2}}}{r a^{2} \mathrm{~d} r \mathrm{~d} \theta} & \frac{\sqrt{1-k r^{2}}}{a^{2} r \sin \theta \mathrm{d} r \mathrm{~d} \phi} \\
\frac{1}{a r \mathrm{~d} \theta \mathrm{d} t} & \frac{\sqrt{1-k r^{2}}}{a^{2} r \mathrm{~d} \theta \mathrm{d} r} & 0 & \frac{1}{a^{2} r^{2} \sin \theta \mathrm{d} \theta \mathrm{d} \phi} \\
\frac{1}{a r \sin \theta \mathrm{d} \phi \mathrm{d} t} & \frac{\sqrt{1-k r^{2}}}{a^{2} r \sin \theta \mathrm{d} \phi \mathrm{d} r} & \frac{1}{a^{2} r^{2} \sin \theta \mathrm{d} \phi \mathrm{d} \theta} & 0
\end{array}\right) \\
\times\left(\begin{array}{cccc}
0 & -\frac{\dot{a}}{\sqrt{1-k r^{2}}} \mathrm{~d} r & -\dot{a} r \mathrm{~d} \theta & -r \dot{a} \sin \theta \mathrm{d} \phi \\
-\frac{\dot{a}}{\sqrt{1-k r^{2}}} \mathrm{~d} r & 0 & \sqrt{1-k r^{2}} \mathrm{~d} \theta & \sin \theta \sqrt{1-k r^{2}} \mathrm{~d} \phi \\
-r \dot{a} \mathrm{~d} \theta & -\sqrt{1-k r^{2}} \mathrm{~d} \theta & 0 & \cos \theta \mathrm{d} \phi \\
-r \dot{a} \sin \theta \mathrm{d} \phi & -\sin \theta \sqrt{1-k r^{2}} \mathrm{~d} \phi & -\cos \theta \mathrm{d} \phi & 0
\end{array}\right)
\end{gathered}
$$

Thus we have 


$$
\left(\begin{array}{cccc}
3 \frac{\dot{a}}{a \mathrm{~d} t} & 2 \frac{\sqrt{1-k r^{2}}}{a r \mathrm{~d} t} & -\frac{\left(1-k r^{2}\right)}{a \mathrm{~d} t \mathrm{~d} r} \mathrm{~d} \theta+\frac{\cos \theta}{a r \sin \theta \mathrm{d} t} & -\frac{\left(1-k r^{2}\right) \sin \theta}{a \mathrm{~d} t \mathrm{~d} r} \mathrm{~d} \phi-\frac{\cos \theta}{a r \mathrm{~d} t \mathrm{~d} \theta} \mathrm{d} \phi \\
-\frac{2 \dot{a} \sqrt{1-k r^{2}}}{a^{2} \mathrm{~d} r} & -\frac{\dot{a}}{a \mathrm{~d} t}-2 \frac{\left(1-k r^{2}\right)}{r a^{2} \mathrm{~d} r} & -\frac{\dot{a} r \sqrt{1-k r^{2}}}{a \mathrm{~d} r \mathrm{~d} t} \mathrm{~d} \theta-\frac{\cos \theta \sqrt{1-k r^{2}}}{r a^{2} \sin \theta \mathrm{d} r}-\frac{r \dot{a} \sin \theta \sqrt{1-k r^{2}}}{a \mathrm{~d} r \mathrm{~d} t} \mathrm{~d} \phi+\frac{\cos \theta \mathrm{d} \phi \sqrt{1-k r^{2}}}{a^{2} r \mathrm{~d} r \mathrm{~d} \theta} \\
-\frac{2 \dot{a}}{a^{2} r \mathrm{~d} \theta} & -\frac{\dot{a}}{a r \sqrt{1-k r^{2}} \mathrm{~d} \theta \mathrm{d} t} \mathrm{~d} r-\frac{\sqrt{1-k r^{2}}}{a^{2} r^{2} \mathrm{~d} \theta} & -\frac{\dot{a}}{a \mathrm{~d} t}+\frac{\left(1-k r^{2}\right)}{a^{2} r \mathrm{~d} r}-\frac{\cos \theta}{a^{2} r^{2} \sin \theta \mathrm{d} \theta} & -\frac{\dot{a} \sin \theta \mathrm{d} \phi}{a \mathrm{~d} \theta \mathrm{d} t}+\frac{\left(1-k r^{2}\right) \sin \theta}{a^{2} r \mathrm{~d} \theta \mathrm{d} r} \mathrm{~d} \phi \\
-2 \frac{\dot{a}}{a^{2} r \sin \theta \mathrm{d} \phi}-\frac{\dot{a}}{a r \sin \theta \sqrt{1-k r^{2}} \mathrm{~d} \phi \mathrm{d} t} \mathrm{~d} r-\frac{\sqrt{1-k r^{2}}}{a^{2} r^{2} \sin \theta \mathrm{d} \phi}-\frac{\dot{a} \mathrm{~d} \theta}{a \sin \theta \mathrm{d} \phi \mathrm{d} t}+\frac{\left(1-k r^{2}\right)}{a^{2} r \sin \theta \mathrm{d} \phi \mathrm{d} r} \mathrm{~d} \theta & -\frac{\dot{a}}{a \mathrm{~d} t}+\frac{\left(1-k r^{2}\right)}{a^{2} r \mathrm{~d} r}+\frac{\cos \theta}{a^{2} r^{2} \sin \theta \mathrm{d} \theta}
\end{array}\right)
$$

Using the fact $\frac{1}{\mathrm{~d} x_{j} \mathrm{~d} x_{k}} \mathrm{~d} x_{i} \equiv 0$ together with (3.11), we obtain

$$
S_{\sigma \gamma}=\left(\begin{array}{cccc}
3 \frac{\dot{a}}{a \mathrm{~d} t} & 2 \frac{\sqrt{1-k r^{2}}}{a r \mathrm{~d} t} & +\frac{\cos \theta}{a r \sin \theta \mathrm{d} t} & 0 \\
-\frac{2 \dot{a} \sqrt{1-k r^{2}}}{a^{2} \mathrm{~d} r} & -\frac{\dot{a}}{a \mathrm{~d} t}-2 \frac{\left(1-k r^{2}\right)}{r a^{2} \mathrm{~d} r} & -\frac{\cos \theta \sqrt{1-k r^{2}}}{r a^{2} \sin \theta \mathrm{d} r} & 0 \\
-\frac{2 \dot{a}}{a^{2} r \mathrm{~d} \theta} & -\frac{\sqrt{1-k r^{2}}}{a^{2} r^{2} \mathrm{~d} \theta} & -\frac{\dot{a}}{a \mathrm{~d} t}+\frac{\left(1-k r^{2}\right)}{a^{2} r \mathrm{~d} r}-\frac{\cos \theta}{a^{2} r^{2} \sin \theta \mathrm{d} \theta} & 0 \\
-2 \frac{\dot{a}}{a^{2} r \sin \theta \mathrm{d} \phi} & -\frac{\sqrt{1-k r^{2}}}{a^{2} r^{2} \sin \theta \mathrm{d} \phi} & 0 & -\frac{\dot{a}}{a \mathrm{~d} t}+\frac{\left(1-k r^{2}\right)}{a^{2} r \mathrm{~d} r}+\frac{\cos \theta}{a^{2} r^{2} \sin \theta \mathrm{d} \theta}
\end{array}\right)
$$

potential matrix is

$$
P_{\sigma \gamma}=S_{\sigma \gamma} \mathrm{d} \varphi_{\sigma \gamma}=\left(\begin{array}{cccc}
0 & 0 & 0 & 0 \\
-\frac{2 \dot{a}}{r \sqrt{1-k r^{2}}} & \frac{2 \dot{a}^{2}}{a^{2}} & 0 & 0 \\
\frac{r \dot{a} \cos \theta}{\sin \theta} & \frac{\cos \theta}{\sin \theta} \sqrt{1-k r^{2}} & 2 \frac{\dot{a}^{2}}{a^{2}}-\frac{\left(1-k r^{2}\right)}{a^{2} r^{2}} & 0 \\
0 & 0 & 0 & 2 \frac{\dot{a}^{2}}{a^{2}}-\frac{\left(1-k r^{2}\right)}{a^{2} r^{2}}
\end{array}\right)
$$

Step3: The components of the Ricci tensor $\hat{R}_{\sigma \gamma}$ in the local frame

$$
\begin{gathered}
\hat{R}_{00}=3 \frac{\ddot{a}}{a} \\
\hat{R}_{11}=\frac{\ddot{a}}{a}+2 \frac{k}{a^{2}}+\frac{2 \dot{a}^{2}}{a^{2}}=\frac{a \ddot{a}+2 k+2 \dot{a}^{2}}{a^{2}} \\
\hat{R}_{22}=\frac{\ddot{a}}{a}+\frac{k}{a^{2}}+\frac{1}{a^{2} r^{2}}+2 \frac{\dot{a}^{2}}{a^{2}}-\frac{\left(1-k r^{2}\right)}{a^{2} r^{2}}=\frac{\left(a \ddot{a}+2 k+2 \dot{a}^{2}\right)}{a^{2}} \\
\hat{R}_{33}=\frac{\ddot{a}}{a}+\frac{k}{a^{2}}+\frac{1}{a^{2} r^{2}}+2 \frac{\dot{a}^{2}}{a^{2}}-\frac{\left(1-k r^{2}\right)}{a^{2} r^{2}}=\frac{\left(a \ddot{a}+2 k+2 \dot{a}^{2}\right)}{a^{2}}
\end{gathered}
$$

Now, we can find the Ricci tensor $R_{\sigma \gamma}$ as follows:

$$
R_{\sigma \gamma}=g_{\sigma \gamma} \hat{R}_{\sigma \gamma}
$$


Then,

$$
R_{\sigma \gamma}=\left(\begin{array}{cccc}
-3 \frac{\ddot{a}}{a} & 0 & 0 & 0 \\
0 & \frac{a \ddot{a}+2 k+2 \dot{a}^{2}}{1-k r^{2}} & 0 & 0 \\
0 & 0 & \left(a \ddot{a}+2 k+2 \dot{a}^{2}\right) r^{2} & 0 \\
0 & 0 & 0 & \left(a \ddot{a}+2 k+2 \dot{a}^{2}\right) r^{2} \sin ^{2} \theta
\end{array}\right)
$$

we can find the Ricci Scalar $R$ as follows:

$$
R=\operatorname{tr}\left(\hat{R}_{\sigma \gamma}\right)=6\left(\frac{a \ddot{a}+2 k+\dot{a}^{2}}{a^{2}}\right)
$$

\subsection{Solution of the Schwarzschild Metric}

EXAMPLE 4

For the Schwarzschild metric

$$
\mathrm{d} s^{2}=-\mathrm{e}^{2 a} \mathrm{~d} t^{2}+\mathrm{e}^{2 b} \mathrm{~d} r^{2}+r^{2} \mathrm{~d} \theta^{2}+r^{2} \sin ^{2} \theta \mathrm{d} \phi^{2}
$$

calculate the components of the Ricci tensor and the Ricci scalar using Abou Layla's methods

SOLUTION 3

we can define the orthonormal vector $\boldsymbol{z}$ as the following

$$
\boldsymbol{z}=\mathrm{e}^{a} \mathrm{~d} t \boldsymbol{L}+\mathrm{e}^{b} \mathrm{~d} r \boldsymbol{i}+r \mathrm{~d} \theta \boldsymbol{j}+r \sin \theta \mathrm{d} \phi \boldsymbol{k}
$$

Now we can calculate the equation of 4-interval angular $\mathrm{d} \vec{\varphi}$ by

$$
\nabla \times \boldsymbol{z}=\left|\begin{array}{cccc}
L & i & j & k \\
\mathrm{e}^{-a} \frac{\partial}{\partial t} & \mathrm{e}^{-b} \frac{\partial}{\partial r} & \frac{1}{r} \frac{\partial}{\partial \theta} & \frac{1}{r \sin \theta} \frac{\partial}{\partial \phi} \\
\mathrm{e}^{a} \mathrm{~d} t & \mathrm{e}^{b} \mathrm{~d} r & r \mathrm{~d} \theta & r \sin \theta \mathrm{d} \phi
\end{array}\right|
$$

then we get

$$
\begin{aligned}
\mathrm{d} \vec{\varphi}= & \nabla \times \boldsymbol{z} \\
= & \left(\mathrm{e}^{-a} \frac{\partial}{\partial t} \mathrm{e}^{b} \mathrm{~d} r-\mathrm{e}^{-b} \frac{\partial}{\partial r} \mathrm{e}^{a} \mathrm{~d} t\right) v^{01} \\
& +\left(\mathrm{e}^{-a} \frac{\partial}{\partial t} r \mathrm{~d} \theta-\frac{1}{r} \frac{\partial}{\partial \theta} \mathrm{e}^{a} \mathrm{~d} t\right) v^{02} \\
& +\left(\mathrm{e}^{-a} \frac{\partial}{\partial t} r \sin \theta \mathrm{d} \phi-\frac{1}{r \sin \theta} \frac{\partial}{\partial \phi} \mathrm{e}^{a} \mathrm{~d} t\right) v^{03} \\
+ & \left(\mathrm{e}^{-b} \frac{\partial}{\partial r} r \mathrm{~d} \theta-\frac{1}{r} \frac{\partial}{\partial \theta} \mathrm{e}^{b} \mathrm{~d} r\right) v^{12} \\
+ & \left(\mathrm{e}^{-b} \frac{\partial}{\partial r} r \sin \theta \mathrm{d} \phi-\frac{1}{r \sin \theta} \frac{\partial}{\partial \phi} \mathrm{e}^{b} \mathrm{~d} r\right) v^{13} \\
+ & \left(\frac{1}{r} \frac{\partial}{\partial \theta} r \sin \theta \mathrm{d} \phi-\frac{1}{r \sin \theta} \frac{\partial}{\partial \phi} r \mathrm{~d} \theta\right) v^{23}
\end{aligned}
$$


Therefore, we have

$$
\begin{aligned}
\mathrm{d} \varphi_{\sigma \gamma}= & \left(\begin{array}{cccc}
0 & -\mathrm{e}^{-b} \frac{\partial}{\partial r} \mathrm{e}^{a} \mathrm{~d} t & 0 & 0 \\
\dot{a} \mathrm{e}^{a-b} \mathrm{~d} r & 0 & \mathrm{e}^{-b} \frac{\partial}{\partial r} r \mathrm{~d} \theta & \mathrm{e}^{-b} \frac{\partial}{\partial r} r \sin \theta \mathrm{d} \phi \\
0 & -\mathrm{e}^{-b} \mathrm{~d} \theta & 0 & \frac{1}{r} \frac{\partial}{\partial \theta} r \sin \theta \mathrm{d} \phi \\
0 & -\mathrm{e}^{-b} \sin \theta \mathrm{d} \phi & -\cos \theta \mathrm{d} \phi & 0 \\
0 & -\dot{a} \mathrm{e}^{a-b} \mathrm{~d} t & 0 & 0 \\
\dot{a} \mathrm{e}^{a-b} \mathrm{~d} t & 0 & \mathrm{e}^{-b} \mathrm{~d} \theta & \mathrm{e}^{-b} \sin \theta \mathrm{d} \phi \\
0 & -\mathrm{e}^{-b} \mathrm{~d} \theta & 0 & \cos \theta \mathrm{d} \phi \\
0 & -\mathrm{e}^{-b} \sin \theta \mathrm{d} \phi & -\cos \theta \mathrm{d} \phi & 0
\end{array}\right)
\end{aligned}
$$

And the components of matrix $\mathrm{d} \bar{\varphi}_{\sigma \gamma}$ will be as the following

$$
\mathrm{d} \bar{\varphi}=\left(\begin{array}{cccc}
0 & -\frac{\mathrm{e}^{-b}}{\mathrm{e}^{a} \mathrm{~d} t} \frac{\partial}{\partial t} & 0 & 0 \\
\frac{\mathrm{e}^{-b}}{\mathrm{e}^{a} \mathrm{~d} t} \frac{\partial}{\partial t} & 0 & \frac{\mathrm{e}^{-b}}{r \mathrm{~d} \theta} \frac{\partial}{\partial r} & \frac{\mathrm{e}^{-b}}{r \sin \theta \mathrm{d} \phi} \frac{\partial}{\partial r} \\
0 & -\frac{\mathrm{e}^{-b}}{r \mathrm{~d} \theta} \frac{\partial}{\partial r} & 0 & \frac{1}{r(r \sin \theta \mathrm{d} \phi)} \frac{\partial}{\partial \theta} \\
0 & -\frac{\mathrm{e}^{-b}}{r \sin \theta \mathrm{d} \phi} \frac{\partial}{\partial r} & -\frac{1}{r(r \sin \theta \mathrm{d} \phi)} \frac{\partial}{\partial \theta} & 0
\end{array}\right)
$$

\section{Step1: The components of the kinetic field matrix $K$}

In our case, we have

$$
\begin{gathered}
F=\mathrm{d} \vec{\varphi} \\
D=\mathrm{d} \bar{\varphi} \\
\tau_{\sigma \gamma}=\frac{1}{z_{\sigma} z_{\gamma}}
\end{gathered}
$$

Then we get

$$
D_{\sigma \gamma}=\left(\begin{array}{cccc}
0 & -\frac{\mathrm{e}^{-b}}{\mathrm{e}^{a} \mathrm{~d} t} \frac{\partial}{\partial t} & 0 & 0 \\
\frac{\mathrm{e}^{-b}}{\mathrm{e}^{a} \mathrm{~d} t} \frac{\partial}{\partial t} & 0 & \frac{\mathrm{e}^{-b}}{r \mathrm{~d} \theta} \frac{\partial}{\partial r} & \frac{\mathrm{e}^{-b}}{r \sin \theta \mathrm{d} \phi} \frac{\partial}{\partial r} \\
0 & -\frac{\mathrm{e}^{-b}}{r \mathrm{~d} \theta} \frac{\partial}{\partial r} & 0 & \frac{1}{r^{2} \sin \theta \mathrm{d} \phi} \frac{\partial}{\partial \theta} \\
0 & -\frac{\mathrm{e}^{-b}}{r \sin \theta \mathrm{d} \phi} \frac{\partial}{\partial r} & -\frac{1}{r^{2} \sin \theta \mathrm{d} \phi} \frac{\partial}{\partial \theta} & 0
\end{array}\right)
$$

we can find the kinetic field matrix in the coordinate basis $(K)$ as 


$$
\begin{aligned}
k_{\sigma \gamma}= & \left(\begin{array}{cccc}
0 & -\frac{\mathrm{e}^{-b}}{\mathrm{e}^{a} \mathrm{~d} t} \frac{\partial}{\partial t} & 0 & 0 \\
\frac{\mathrm{e}^{-b}}{\mathrm{e}^{a} \mathrm{~d} t} \frac{\partial}{\partial t} & 0 & \frac{\mathrm{e}^{-b}}{r \mathrm{~d} \theta} \frac{\partial}{\partial r} & \frac{\mathrm{e}^{-b}}{r \sin \theta \mathrm{d} \phi} \frac{\partial}{\partial r} \\
0 & -\frac{\mathrm{e}^{-b}}{r \mathrm{~d} \theta} \frac{\partial}{\partial r} & 0 & \frac{1}{r^{2} \sin \theta \mathrm{d} \phi} \frac{\partial}{\partial \theta} \\
0 & -\frac{\mathrm{e}^{-b}}{r \sin \theta \mathrm{d} \phi} \frac{\partial}{\partial r} & -\frac{1}{r^{2} \sin \theta \mathrm{d} \phi} \frac{\partial}{\partial \theta} & 0
\end{array}\right) \\
\times & \left(\begin{array}{cccc}
0 & -\dot{a} \mathrm{e}^{a-b} \mathrm{~d} t & 0 & 0 \\
\dot{a} \mathrm{e}^{a-b} \mathrm{~d} t & 0 & \mathrm{e}^{-b} \mathrm{~d} \theta & \mathrm{e}^{-b} \sin \theta \mathrm{d} \phi \\
0 & -\mathrm{e}^{-b} \mathrm{~d} \theta & 0 & \cos \theta \mathrm{d} \phi \\
0 & -\mathrm{e}^{-b} \sin \theta \mathrm{d} \phi & -\cos \theta \mathrm{d} \phi & 0
\end{array}\right)
\end{aligned}
$$

The nonzero components of $k_{\sigma \gamma}$ matrix are

$$
\begin{aligned}
& k_{00}=-\frac{\mathrm{e}^{-b}}{\mathrm{e}^{a} \mathrm{~d} t} \frac{\partial}{\partial r}\left(\dot{a} \mathrm{e}^{a-b} \mathrm{~d} t\right)=-\ddot{a} \mathrm{e}^{-2 b}-\dot{a}^{2} \mathrm{e}^{-2 b}+\dot{a} \dot{b} \mathrm{e}^{-2 b} \\
& k_{11}=\frac{\mathrm{e}^{-b}}{\mathrm{e}^{a} \mathrm{~d} t} \frac{\partial}{\partial r}\left(-\dot{a} \mathrm{e}^{a-b} \mathrm{~d} r\right)+\frac{\mathrm{e}^{-b}}{r \mathrm{~d} \theta} \frac{\partial}{\partial r}\left(-\mathrm{e}^{-b} \mathrm{~d} \theta\right)+\frac{\mathrm{e}^{-b}}{r \sin \theta \mathrm{d} \phi} \frac{\partial}{\partial r}\left(-\mathrm{e}^{-b} \sin \theta \mathrm{d} \phi\right) \\
& k_{11}=-\ddot{a} e^{-2 b}-\dot{a}^{2} \mathrm{e}^{-2 b}+\dot{a} \dot{b} \mathrm{e}^{-2 b}+2 \frac{\dot{b}}{r} \mathrm{e}^{-2 b} \\
& k_{22}=-\frac{e^{-b}}{r \mathrm{~d} \theta} \frac{\partial}{\partial r}\left(\mathrm{e}^{-b} \mathrm{~d} \theta\right)+\frac{1}{r^{2} \sin \theta \mathrm{d} \phi} \frac{\partial}{\partial \theta}(-\cos \theta \mathrm{d} \phi) \\
& k_{22}=\frac{\dot{b}}{r} \mathrm{e}^{-2 b}+\frac{1}{r^{2}} \\
& k_{33}=-\frac{\mathrm{e}^{-b}}{r \sin \theta \mathrm{d} \phi} \frac{\partial}{\partial r}\left(\mathrm{e}^{-b} \sin \theta \mathrm{d} \phi\right)-\frac{1}{r^{2} \sin \theta \mathrm{d} \phi} \frac{\partial}{\partial \theta}(\cos \theta \mathrm{d} \phi) \\
& k_{33}=\frac{\dot{b}}{r} \mathrm{e}^{-2 b}+\frac{1}{r^{2}}
\end{aligned}
$$

Step2: The components of the potential field matrix $P$

$$
\tau_{\sigma \gamma}=\frac{1}{z_{\sigma} z_{\gamma}}=\left(\begin{array}{cccc}
0 & \frac{1}{\mathrm{e}^{a} \mathrm{e}^{b} \mathrm{~d} t \mathrm{~d} r} & \frac{1}{r \mathrm{e}^{a} \mathrm{~d} t \mathrm{~d} \theta} & \frac{1}{r \mathrm{e}^{a} \sin \theta \mathrm{d} t \mathrm{~d} \phi} \\
\frac{1}{\mathrm{e}^{a} \mathrm{e}^{b} \mathrm{~d} t \mathrm{~d} r} & 0 & \frac{1}{r \mathrm{e}^{b} \mathrm{~d} r \mathrm{~d} \theta} & \frac{1}{r \mathrm{e}^{b} \sin \theta \mathrm{d} r \mathrm{~d} \phi} \\
\frac{1}{r \mathrm{e}^{a} \mathrm{~d} \theta \mathrm{d} t} & \frac{1}{r \mathrm{e}^{b} \mathrm{~d} \theta \mathrm{d} r} & 0 & \frac{1}{r^{2} \sin \theta \mathrm{d} \theta \mathrm{d} \phi} \\
\frac{1}{r \mathrm{e}^{a} \sin \theta \mathrm{d} \phi \mathrm{d} t} & \frac{1}{r \mathrm{e}^{b} \sin \theta \mathrm{d} \phi \mathrm{d} r} & \frac{\sin \theta}{r^{2} \sin \theta \mathrm{d} \phi \mathrm{d} \theta} & 0
\end{array}\right)
$$

The square of vector $\mathrm{d} \vec{\varphi}$ form as, 


$$
\begin{aligned}
(\mathrm{d} \varphi)^{2} & =\left(\begin{array}{cccc}
0 & -\dot{a} \mathrm{e}^{a-b} \mathrm{~d} t & 0 & 0 \\
\dot{a} \mathrm{e}^{a-b} \mathrm{~d} t & 0 & \mathrm{e}^{-b} \mathrm{~d} \theta & \mathrm{e}^{-b} \sin \theta \mathrm{d} \phi \\
0 & -\mathrm{e}^{-b} \mathrm{~d} \theta & 0 & \cos \theta \mathrm{d} \phi \\
0 & -\mathrm{e}^{-b} \sin \theta \mathrm{d} \phi & -\cos \theta \mathrm{d} \phi & 0
\end{array}\right) \\
& \times\left(\begin{array}{cccc}
0 & -\dot{a} \mathrm{e}^{a-b} \mathrm{~d} t & 0 & 0 \\
\dot{a} \mathrm{e}^{a-b} \mathrm{~d} t & 0 & \mathrm{e}^{-b} \mathrm{~d} \theta & \mathrm{e}^{-b} \sin \theta \mathrm{d} \phi \\
0 & -\mathrm{e}^{-b} \mathrm{~d} \theta & 0 & \cos \theta \mathrm{d} \phi \\
0 & -\mathrm{e}^{-b} \sin \theta \mathrm{d} \phi & -\cos \theta \mathrm{d} \phi & 0
\end{array}\right)
\end{aligned}
$$

thus

$$
\begin{aligned}
& F^{2}=(\mathrm{d} \varphi)^{2} \\
& =\left(\begin{array}{cccc}
0 & 0 & -\dot{a} \mathrm{e}^{a-2 b} \mathrm{~d} t \mathrm{~d} \theta & -\dot{a} \mathrm{e}^{a-2 b} \sin \theta \mathrm{d} t \mathrm{~d} \phi \\
0 & 0 & 0 & \mathrm{e}^{-b} \cos \theta \mathrm{d} \theta \mathrm{d} \phi \\
-\dot{a} \mathrm{e}^{a-2 b} \mathrm{~d} \theta \mathrm{d} t & 0 & 0 & -\mathrm{e}^{-2 b} \sin \theta \mathrm{d} \theta \mathrm{d} \phi \\
-\dot{a} \mathrm{e}^{a-2 b} \sin \theta \mathrm{d} \phi \mathrm{d} t & \cos \theta \mathrm{e}^{-b} \mathrm{~d} \phi \mathrm{d} \theta & -\mathrm{e}^{-2 b} \sin \theta \mathrm{d} \phi \mathrm{d} \theta & 0
\end{array}\right)
\end{aligned}
$$

Using (3.12) the matrix of potential field matrix $P_{\sigma \gamma}$ will be

$$
P_{\sigma \gamma}=\left(\begin{array}{cccc}
-2 \frac{\dot{a}}{r} \mathrm{e}^{-2 b} & 0 & 0 & 0 \\
0 & 0 & 0 & 0 \\
0 & 0 & -\frac{\dot{a}}{r} \mathrm{e}^{-2 b}-\frac{1}{r^{2}} \mathrm{e}^{-2 b} & 0 \\
0 & 0 & 0 & -\frac{\dot{a}}{r} \mathrm{e}^{-2 b}-\frac{1}{r^{2}} \mathrm{e}^{-2 b}
\end{array}\right)
$$

\section{Step3: The components of the Ricci tensor}

$$
\hat{R}_{\sigma \gamma}=k_{\sigma \gamma}+P_{\sigma \gamma}
$$

Then we get

$$
\begin{gathered}
\hat{R}_{00}=-\ddot{a} \mathrm{e}^{-2 b}-\dot{a}^{2} \mathrm{e}^{-2 b}+\dot{a} \dot{b} \mathrm{e}^{-2 b}-2 \frac{\dot{a}}{r} \mathrm{e}^{-2 b} \\
\hat{R}_{11}=-\ddot{a} \mathrm{e}^{-2 b}-\dot{a}^{2} \mathrm{e}^{-2 b}+\dot{a} \dot{b} \mathrm{e}^{-2 b}+2 \frac{\dot{b}}{r} \mathrm{e}^{-2 b} \\
\hat{R}_{22}=\frac{\dot{b}}{r} \mathrm{e}^{-2 b}+\frac{1}{r^{2}}-\frac{\dot{a}}{r} \mathrm{e}^{-2 b}-\frac{1}{r^{2}} \mathrm{e}^{-2 b} \\
\hat{R}_{33}=\frac{\dot{b}}{r} \mathrm{e}^{-2 b}+\frac{1}{r^{2}}-\frac{\dot{a}}{r} \mathrm{e}^{-2 b}-\frac{1}{r^{2}} \mathrm{e}^{-2 b}
\end{gathered}
$$

Now, we can find the Ricci tensor $R_{\sigma \gamma}$ as follows:

$$
R_{\sigma \gamma}=g_{\sigma \gamma} \hat{R}_{\sigma \gamma}
$$

Then,

$$
\begin{gathered}
R_{00}=\ddot{a} \mathrm{e}^{2 a-2 b}+\dot{a}^{2} \mathrm{e}^{2 a-2 b}-\dot{a} \dot{b} \mathrm{e}^{2 a-2 b}+\frac{2}{r} \dot{a} \mathrm{e}^{2 a-2 b} \\
R_{11}=-\ddot{a}-\dot{a}^{2}+\dot{a} \dot{b}+\frac{2}{r} \dot{b}
\end{gathered}
$$




$$
\begin{gathered}
R_{22}=r \dot{b} \mathrm{e}^{-2 b}+1-r \dot{a} \mathrm{e}^{-2 b}-\mathrm{e}^{-2 b} \\
R_{33}=r \sin ^{2} \theta \dot{b} \mathrm{e}^{-2 b}+\sin ^{2} \theta-r \dot{a} \mathrm{e}^{-2 b} \sin ^{2} \theta-\mathrm{e}^{-2 b} \sin ^{2} \theta
\end{gathered}
$$

The Ricci Scalar $R$ can be found by calculating $\operatorname{tr}\left(\hat{R}_{\sigma \gamma}\right)$ :

$$
R=\operatorname{tr}\left(\hat{R}_{\sigma \gamma}\right)=-2 \frac{\ddot{a}+\dot{a}^{2}-\dot{a} \dot{b}}{\mathrm{e}^{2 b}}-4 \frac{\dot{a}-\dot{b}}{r \mathrm{e}^{2 b}}+2 \frac{\mathrm{e}^{2 b}-1}{r^{2} \mathrm{e}^{2 b}}
$$

\section{Conclusion}

Dealing with general relativity theory in a matrix form will ease the way to find the Ricci tensor, Ricci scalar, and Einstein Field Equation. The most important, it will open the doors to unify this theory with electromagnetic theory in the near future.

\section{Acknowledgements}

I would like have the opportunity to obtain the Canadian citizenship in the near future due to the current conditions in my city.

I'll be thankful to Canadian authorities if they help me in this issue, especially it will help in publishing of more of my scientific research.

\section{Conflicts of Interest}

The author declares no conflicts of interest regarding the publication of this paper.

\section{References}

[1] Abou Layla, A.K. (2018) Old Mechanics, Gravity, Electromagnetics and Relativity in One Theory: Part I. Journal of High Energy Physics, Gravitation and Cosmology, 4, 529-540. https://doi.org/10.4236/jhepgc.2018.43031

[2] Wikipedia, General Relativity. https://en.wikipedia.org/wiki/General_relativity 